\title{
Broad-band polarization-insensitive all-dielectric metalens enabled by intentional off-resonance waveguiding at mid-wave infrared
}

\author{
Cite as: Appl. Phys. Lett. 114, 043105 (2019); doi: 10.1063/1.5063967 \\ Submitted: 03 October 2018 - Accepted: 15 January 2019 - Published Online: \\ 01 February 2019
}
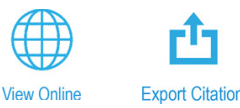

Ibrahim Tanriover ${ }^{\top}$ and Hilmi Volkan Demir ${ }^{1,2,3, a)}$

\author{
AFFILIATIONS \\ ${ }^{\top}$ Department of Electrical and Electronics and UNAM-Institute of Material Science and Nanotechnology, Bilkent University, \\ TR-06800 Ankara, Turkey \\ ${ }^{2}$ Department of Physics, Bilkent University, TR-06800 Ankara, Turkey \\ ${ }^{3}$ LUMINOUS! Centre of Excellence for Semiconductor Lighting and Displays, School of Electrical and Electronic Engineering, \\ School of Physical and Mathematical Sciences, School of Material Science and Nanotechnology, Nanyang Technological \\ University, 50 Nanyang Avenue, 639798 Singapore \\ a) Electronic mail: volkan@stanfordalumni.org
}

\begin{abstract}
Metasurfaces are promising candidates to take the place of conventional optical components as they enable wavefront engineering at sub- and near-wavelength distances along both lateral and vertical directions. Plasmonic metasurfaces containing sub-wavelength metallic structures constitute initial examples of this concept. However, plasmonic metasurfaces cannot achieve satisfactory efficiencies in the transmission mode due to their intrinsic losses. The low efficiencies of transmissive plasmonic metasurfaces motivated solutions using dielectric ones. Such high-efficiency all dielectric metasurfaces depend on either resonance tuning or Pancharatnam-Berry (geometrical) phase approaches. However, these approaches are limited to either narrow operation bands or suffer polarization dependency. Here, we propose and show high-index dielectric nanopillars operated as cylindrical waveguides deliberately in the off-resonance regime to achieve polarization independent wavefront control over wide spectral bands. As a proof-of-concept structure, we demonstrated a focusing metalens operating at wavelengths from 4.0 to $4.6 \mu \mathrm{m}$ under both s- and p-polarized illuminations. The designed lens maintains the focusing operation with a maximum of $4 \%$ focal distance shift having a relative efficiency of $>94 \%$ and an absolute efficiency of $>67 \%$ all over the defined spectral band of $600 \mathrm{~nm}$, which outperforms the previously reported metalenses in terms of wide-band operation with high performance.
\end{abstract}

Published under license by AIP Publishing. https://doi.org/10.1063/1.5063967

Metasurfaces have emerged conceptually as one of the most promising structures to replace bulky optics. By making it possible to manipulate light at sub-wavelength thicknesses, metasurfaces provide us with the ability to overcome the size limitations of various applications, for example, in wearable and portable devices. ${ }^{26}$ Based on metasurfaces, various optical components including polarizers, holograms, beam deflectors, and lenses have been previously reported. ${ }^{1-23}$ These components were obtained via controlling the wavefront at sub-wavelength distances along both lateral and vertical directions. Precursor examples of metasurfaces used plasmonic structures. ${ }^{10,13,14}$ However, these suffered from fundamental ohmic losses resulting in limited efficiency.
Recently, to avoid losses, all-dielectric metasurfaces have been introduced, which have led to high levels of efficiencies. $^{1-9,11,12,15-17,19-23}$ In these, there have been basically two main approaches taken to obtain a full control of the wavefront via dielectric structures. One of them is based on the resonance tuning, which has been employed in most of the earlier dielectric metasurfaces. ${ }^{12,15,21,22}$ However, the operation of these structures is, in general, limited to narrow spectral bands. Although there are some multi-wavelength designs proposed to overcome this limitation, they work only for a few specific discrete wavelengths and do not exhibit the desired continuous wideband operation. ${ }^{7}$ The other approach is based on utilizing phase differences via the Pancharatnam-Berry (PB) phase..$^{3,4,8-10,23}$ In 
this approach, broad-band phase responses can be obtained. However, this is limited to circularly polarized light. As a result, a polarization-insensitive solution exhibiting broad-band performance still lacks to date.

Here, to address this need, we propose a class of dielectric metasurfaces enabled by the waveguiding of their unit cells intentionally operated in the off-resonance regime and demonstrate a proof-of-concept dielectric metalens working in the mid-wave infrared band that exhibits both polarization-insensitive and broad-band operation. Here, we show that, by deliberately working off-resonance, such a full control of the wavefront over a broad spectral band can be achieved via the guiding. As a direct result of spherical symmetry of the unit cells (and thus the resulting metasurface), we obtain the complete polarization independency in our dielectric metalens over the entire defined spectral band.

For a working model as the metasurface unit cell, we used a single step-index cylindrical waveguide in the form of a high enough nanopillar made of $\mathrm{Si}$, where the core is $\mathrm{Si}$ and the cladding is the surrounding medium (air in this case). Here, since the field confinement occurs inside the high refractive index core, keeping the distance between pillars large enough ensures uncoupled operation for all pillars, as seen in the supplementary material. This allows us to assume the cladding radius to be infinite and the field at the cladding borders to diminish. Making these assumptions, we numerically solved the resulting cylindrical waveguide equation $^{24}$ and compared the results to observe the dominance of waveguiding over other optical effects in terms of the phase response. For simplicity, all pillars were structured to support only the fundamental mode $\left(\mathrm{HE}_{11}\right)$. The phase response of a waveguide is calculated as

$$
\phi_{\mathrm{WG}}=\frac{2 \pi}{\lambda} n_{\text {eff }}\left(\mathrm{R}, \lambda, n_{\text {cladding }}, n_{\text {core }}\right) h,
$$

where $h$ is the height of nanopillars, $n_{\text {eff }}$ is the effective index of refraction, and $\lambda$ is the operation wavelength. The agreement with the numerical solution of the resulting waveguide equation (using MATLAB) and finite difference time domain (FDTD) simulations is shown in Fig. 1(a). We also denote that the field confinement inside the pillars increases with the increasing radius, which in turn increases the dominance of guiding on overall response.

In wavefront engineering, the relative phase response of the unit cells is the point of interest. For instance, to make a focusing flat lens, the phase response that should be introduced by each nanopillar is

$$
\phi_{\text {req }}(r)=-\frac{2 \pi}{\lambda}\left(\sqrt{r^{2}+f^{2}}-f\right),
$$

where $f$ is the focal length, $\lambda$ is the wavelength, and $r$ is the distance from the center. Equating (1) and (2) for two arbitrary pillars on a flat lens leads to

$$
-\left(\sqrt{r_{1}^{2}+f^{2}}-\sqrt{r_{2}^{2}+f^{2}}\right)=h \Delta n_{e f f}\left(R_{1}, R_{2}, \lambda\right),
$$

where $\Delta n_{\text {eff }}\left(R_{1}, R_{2}, \lambda\right)=n_{\text {eff }}\left(R_{1}, \lambda\right)-n_{\text {eff }}\left(R_{2}, \lambda\right), r_{1}$ and $r_{2}$ are the locations, and $R_{1}$ and $R_{2}$ are the radii of the two arbitrary nanopillars. As the left-hand side of Eq. (3) is constant, the right-hand (a)

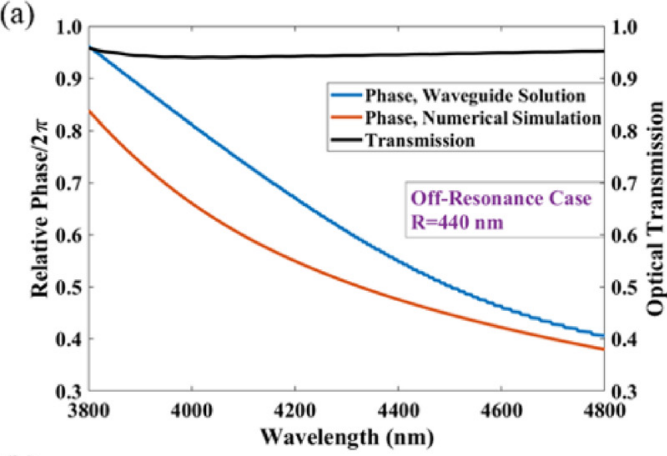

(b)

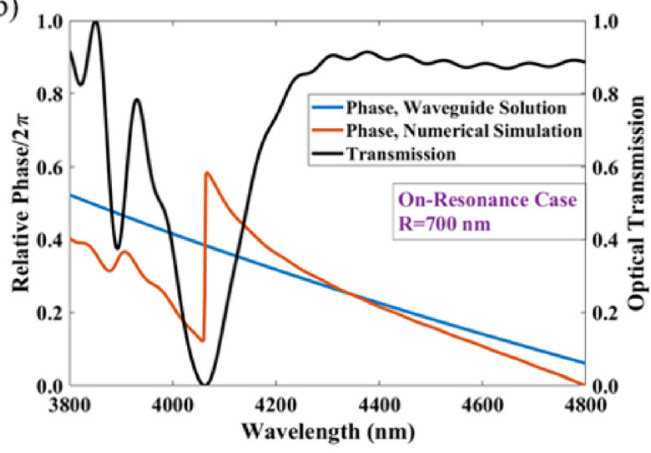

(c)

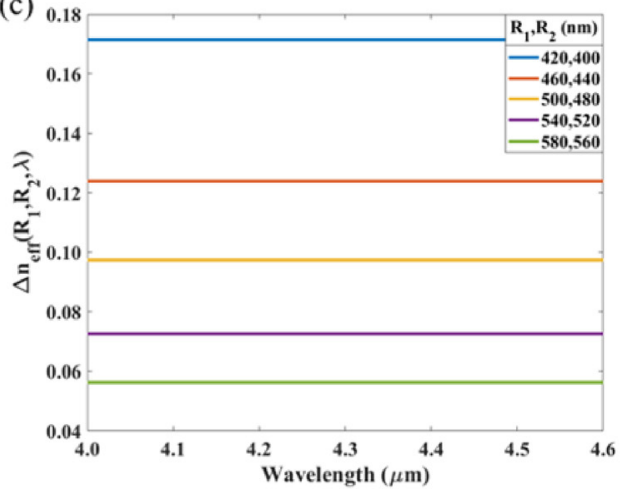

FIG. 1. (a) Transmission and phase response of a cylindrical nanopillar with radius $\mathrm{R}=440 \mathrm{~nm}$ operating in the off-resonance regime. (b) Transmission and phase response of a cylindrical nanopillar with radius $R=700 \mathrm{~nm}$ operating in the resonance regime. The transmission was obtained using the FDTD method. The phase responses were obtained by both numerically solving the cylindrical waveguide equation and FDTD simulations. (c) Effective index difference between arbitrary waveguides in our design spectral band.

side must also be constant over the targeted spectral range to secure perfectly achromatic operation. As seen from Fig. 1(c), cylindrical waveguides almost satisfy this condition.

For a proof-of-concept demonstration of the metasurface, we continued with $\mathrm{Si}$ as the core material. There are two important properties that lead us to make this choice, apart from silicon's general and well-known advantages. One of them is its high index of refraction in our spectral band of interest. As seen from Eq. (1), we have two variables to manipulate the phase response of a waveguide. The first one is the height (h) and the 
second one is $n_{\text {eff. }}$. The height allows us to linearly control the phase difference between pillars and the phase range that we can cover. However, increasing $h$ increases the aspect ratio and, as a result, decreases the feasibility of fabricating this nanostructure. Therefore, the variations of $n_{\text {eff }}$ are kept large enough to make $h$ reasonable. This effective index is a function of the radius of the waveguide, the operation wavelength, and the refractive indices of both the core and the cladding. Its range is between the refractive index of the surrounding medium and the refractive index of the core material. As a result, a higher index contrast between the core and the cladding results in a larger difference in the phase response of different nanopillars. The other reason behind our material choice is the spectral position of resonances. Using $\mathrm{Si}$, we can obtain off-resonance operation for a wide enough range of pillar radii and heights, which allows us to acquire the desired phase responses across our design band.

Working opportunely in the off-resonance regime is one of the key elements of our approach. There are several structures reported having similar geometry with ours, some of which do not report the guiding effect. ${ }^{2,5}$ However, all of these previous structures possess only narrow operation bands contrary to ours. The reason behind this contrast is the resonances. These previous reports use the resonance regime as all of them employed the resonance tuning approach. As seen from Fig. 1(b), resonances disrupt broad-band waveguiding by causing discontinuities at the phase response and lead to abrupt changes in the transmission.

To shape the wavefront, we designed a set of transmissive unit cells all having the same thickness. The phase shift introduced by these unit cells spans the entire range of 0 to $2 \pi$. Here, the unit cells were designed as cylindrical silicon nanopillars on top of a glass $\left(\mathrm{SiO}_{2}\right)$ substrate [Fig. 2(a)]. The height $h$ of pillars was set to be $2 \mu \mathrm{m}$, which is half of the lowest border of the operating wavelength range. Phase responses of the pillars were obtained (a)

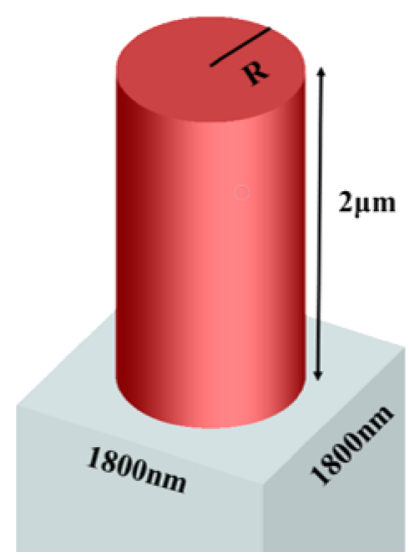

(b)

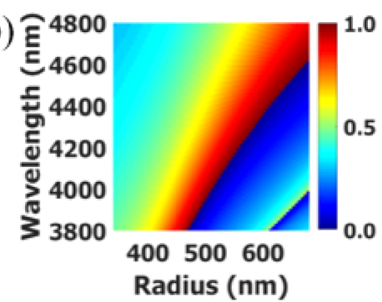

(c)

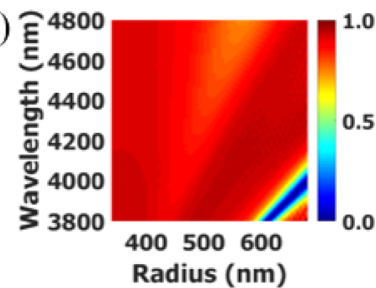

FIG. 2. (a) Side view of a unit cell: a Si nanopillar on top of a glass substrate. The substrate thickness taken to be infinite as it would be much thicker than the nanopillar height, $h=2 \mu \mathrm{m}$. The radius of nanopilars changes from 340 to $680 \mathrm{~nm}$, and the side length of the square lattice is $1800 \mathrm{~nm}$. (b) Phase response of the nanopillars versus wavelength and radius. (c) Transmission of unit cells versus wavelength and radius. The very low transmission values at shorter wavelengths for large radius values show the resonant areas. by tuning the radius $R$. As our approach relies on the offresonance regime, we chose a non-resonant range for $\mathrm{R}$ using the approximate Mie resonance condition $\left(\frac{\lambda}{2 n} q \approx R\right),{ }^{25}$ where $n$ is the refractive index of silicon, $\lambda$ is the borders of our spectral band of operation, and $q$ is an integer. We numerically solved the cylindrical waveguide equation for this range of $\mathrm{R}$ to obtain the first estimation of our radius set. Then, we performed finitedifference time-domain simulations (Lumerical FDTD Solutions) for the pre-determined $\mathrm{R}$ values to eliminate resonant ones and reach the final design set. The overall geometry of our unit cells is illustrated in Fig. 2(a). We have square unit cells with an edge length of $1.8 \mu \mathrm{m}$. The thickness of the $\mathrm{SiO}_{2}$ layer was taken infinitely long (as it is much thicker than the pillar height in practice). The radius of Si nanopillars varies from 340 to $680 \mathrm{~nm}$. The phase and transmission responses of the nanopillars obtained via FDTD simulations are shown in Figs. 2(b) and 2(c), respectively. As seen in the figure, these unit cells are highly transmissive and introduce a full relative phase shift covering the whole $0-2 \pi$ range for the entire spectral band of 4.0 to $4.6 \mu \mathrm{m}$. The low transmission values in the right bottom corner of Fig. 2(c) represent the resonant regime, which is out of our operating band.

The ability to spatially manipulate the phase of light enables us to shape the wavefront of light. By wavefront engineering, many optical components such as beam deflectors and converging and diverging lenses can be designed. Here, for a proof-ofconcept demonstration, we designed a micro-lens operating in the spectral range of 4.0 to $4.6 \mu \mathrm{m}$ to indicate validity of our approach as a complete design. The lens was designed to have a focal length of $48 \mu \mathrm{m}$ as this is in the far field and a reasonable value for applications including focal plane arrays (FPAs) and various types of infrared sensors.

The top view of the lens is shown in Fig. 3(a). The nanopillars are located on nested circles, where the radius values of the (a)

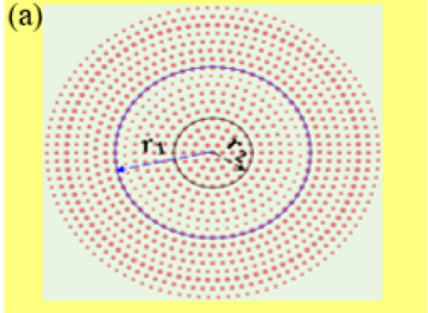

(c)

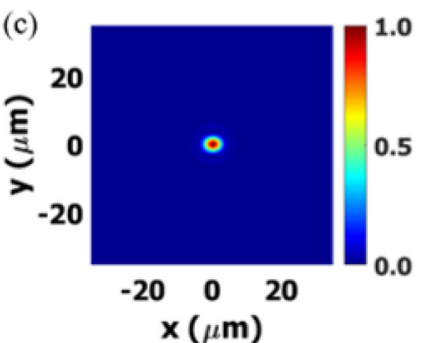

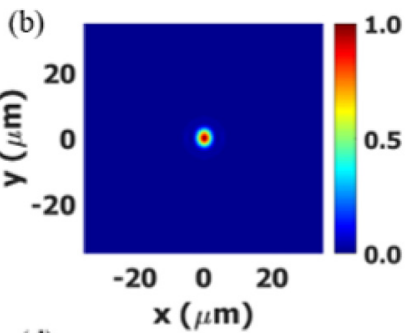

(b)

(d)

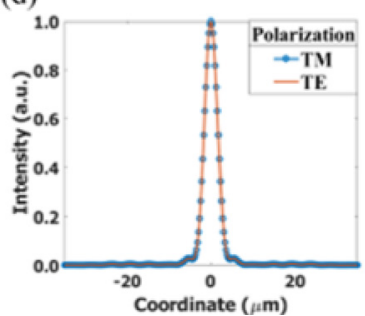

FIG. 3. (a) Top view of the lens with a $35 \mu \mathrm{m}$ radius. (b) and (c) Light distribution at the focal plane ( $\mathrm{z}=48 \mu \mathrm{m}$ plane) of metalens under $4.2 \mu \mathrm{m}$ TM and TE polarized light, respectively. (d) Horizontal cut of the focal spot under TE and TM illumination. All intensities are normalized. 

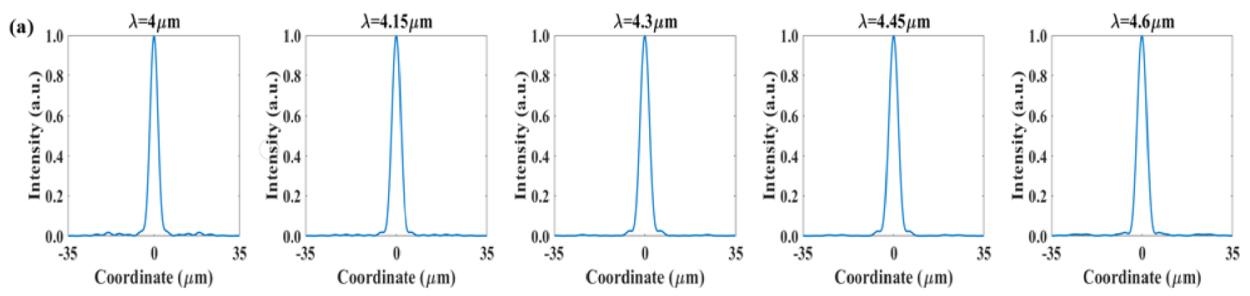

(b)

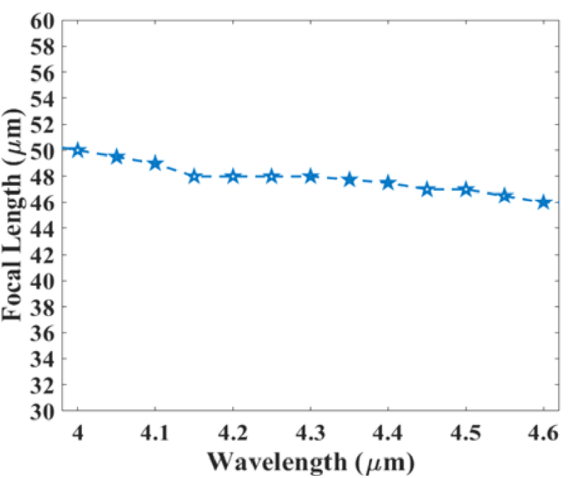

(c)

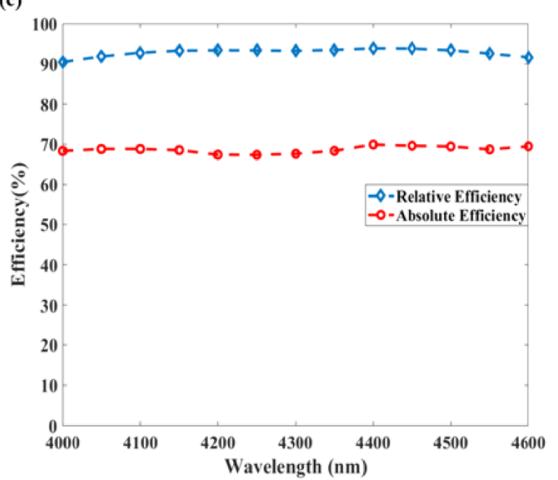

FIG. 4. (a) Horizontal cuts of the focal plane under illumination at 4.00, 4.15, $4.30,4.45$, and $4.60 \mu \mathrm{m}$. (b) Focal distances of the lens in the spectral band of operation. (c) Relative and absolute efficiencies of the lens in the spectral band of operation. The efficiency values calculated at $48 \mu \mathrm{m}$, which is the focal length at the design wavelength. consecutive circles differ by $1.8 \mu \mathrm{m}$, and the distance between the consecutive pillars on the same circle is also $1.8 \mu \mathrm{m}$. The required position dependent phase response of a flat lens is given by Eq. (2). The distance from the center $r$ is the radius of the circles in this case. As a result, all pillars forming the same circle are identical.

The focusing behavior at our design wavelength $(4.2 \mu \mathrm{m})$ can be seen from various perspectives in Fig. 3. Figures 3(a) and 3(b) show the focal planes of the lens under TM and TE illuminations, respectively, and Fig. 3(c) depicts horizontal cuts of these planes. As seen from both the focal planes and their horizontal cuts, the focal spot is identical in the main lobe for the two perpendicular polarizations, indicating the polarization insensitivity. The full-width-half-maximum (FWHM) value is found to be $0.40 \lambda$, where the diffraction limit is calculated as $0.36 \lambda$. The focal spot radius is determined as $4.0 \mu \mathrm{m}$, which is approximately twice of the FWHM. The obtained focusing efficiency, which is calculated as the ratio of the total light intensity at the focal spot to the total transmitted light, is $94.0 \%$. The absolute efficiency, which is defined as the ratio of the total light intensity at the focal spot to the incident light intensity, is found as $67.7 \%$.

The spectral operation band is defined as the wavelength range within which the lens maintains focusing behavior with $<5 \%$ decrease in efficiency and $<5 \%$ shift of focal distance. Figure 4(a) shows the horizontal cuts of the focal plane at various wavelengths in the operating band, indicating the permanency of the focusing behavior. There are two main parameters to determine the broad-band performance of a focusing lens: the change of the focal length and the change in the focusing efficiency. As seen in Fig. 4(b), the focal length remains constant for an almost $600 \mathrm{~nm}$ of wavelength span around the design wavelength, and the maximum focal shifts are $\pm 2 \mu \mathrm{m}$. The efficiency values are calculated at the focal distance of the design wavelength. As seen from Fig. 4(c), the relative efficiency is always higher than $90 \%$ and the absolute efficiency is almost $70 \%$. Both efficiencies only slightly change across the entire operating band.

TABLE I. Performance metrics of the previously reported metalenses and their comparison with this work.

\begin{tabular}{|c|c|c|c|c|c|c|}
\hline & $\frac{\text { FWHM spot size }(\lambda)}{\text { Diffraction limit }(\lambda)}$ & Focusing efficiency & Polarization & Wavelength (nm) & Aspect ratio & Numerical aperture \\
\hline Reference $2^{a}$ & $0.51 / 0.5$ & $\sim 52 \%$ & Insensitive & 1550 & 4.7 & 0.97 \\
\hline Reference $3^{b}$ & $\sim 0.7 / 0.625$ & $86 \%$ & Circular & 405 & 3.86 & 0.8 \\
\hline Reference $4^{b}$ & $\sim 0.255 / 2.5$ & $20 \%$ & Circular & 500 & $\sim 2.8$ & 0.2 \\
\hline Reference $5^{\mathrm{b}}$ & $\sim 0.64 / 0.6$ & $60 \%$ & Insensitive & 660 & $\sim 2.7$ & 0.85 \\
\hline Reference $6^{a}$ & $1.56 / 1.33$ & $80 \%$ & Insensitive & 3200 & $\sim 2.2$ & 0.2 \\
\hline Reference $7^{a}$ & $1.94 / 1.72$ & $\sim 72 \%$ & Insensitive & 1550 & 7 & 0.29 \\
\hline Reference $8^{b}$ & $18.7 / 6.25$ & $10 \%$ & Not Reported & 1550 & 5.3 & 0.04 \\
\hline Reference $9^{b}$ & $5.35 / 4.64$ & $\sim 42 \%$ & Circular & 560 & 17.8 & 0.106 \\
\hline This work ${ }^{a}$ & $\sim 0.4 / 0.36$ & $68 \%$ & Insensitive & 4200 & $\sim 3$ & 0.73 \\
\hline
\end{tabular}

asimulation result.

${ }^{\mathrm{b}}$ Measurement result. 
TABLE II. Figure of merits (FoMs) defined to quantitatively analyze broad-band performance. Here, $\mathrm{F}$ is the focal length, $\lambda$ is the operating wavelength, and $\eta$ is the percentage efficiency.

\begin{tabular}{lc}
\hline \hline FoM $1\left(\% \mathrm{~nm}^{-1}\right)$ & {$\left[\Delta \mathrm{F} /\left(\mathrm{F}^{*} \Delta \lambda\right)\right] \times 100$} \\
FoM $2\left(\% \mathrm{~nm}^{-1}\right)$ & $\Delta \eta / \Delta \lambda$ \\
FoM 3 $\left(\% \mathrm{~nm}^{-1}\right)$ & {$[(\Delta \eta / \eta) / \Delta \lambda] \times 100$} \\
\hline \hline
\end{tabular}

In Table I, we compared the performance of the proposed structure with the previous reports, in terms of the typical performance metrics. Some of the values given in the table belong to broad-band or multi-wavelength designs while the rest belong to single-wavelength designs. We chose the wavelength with the highest efficiency value for multi-wavelength designs and the values obtained for the central wavelength for the broad-band designs. In practice, a slight decrease in FWHM and a $5 \%-10 \%$ decrease in absolute efficiency may emerge due to possible fabrication imperfections. As the field is highly confined in pillars and the distance between the adjacent pillars is far enough, it is not expected to observe a decrease in the performance resulting from mutual coupling.

The broad-band performance of a focusing lens can be evaluated from two major points of view. These are the shift of the focal distance and change in the focusing efficiency with respect to the variation in the operating wavelength. To quantitatively compare our proposed structure with the achromatic designs and similar geometries reported in the literature, we defined three figure of merits (FoMs) in Table II, each of which underlines different aspects of the broad-band focusing behavior. To analytically evaluate the achromaticity from the focal shift perspective, FoM1 is defined as the percentage shift of the focal distance per change in the operating wavelength, which is ideally equal to zero for perfect achromatic lenses. To evaluate the efficiency aspect of achromaticity, we defined FoM2 and FoM3. The former only considers the change in efficiency with respect to the change in the operating wavelength. The latter also takes into account the efficiency at the design wavelength, which is an important performance metric but is not directly related to the achromaticity. As it can be directly understood from the definitions of these FoMs, lower values indicate better performance. The calculated FoMs for different designs are summarized in Table III. As seen in the table, our design outperforms all of these previous reports in terms of both the focal distance shift and the efficiency change.

TABLE III. Comparison of previous reports and this work in terms of the defined FoMs.

\begin{tabular}{lccc}
\hline \hline & FoM $1\left(\% \mathrm{~nm}^{-1}\right)$ & FoM 2 $\left(\% \mathrm{~nm}^{-1}\right)$ & FoM 3 $\left(\% \mathrm{~nm}^{-1}\right)$ \\
\hline Reference $2^{\mathrm{a}}$ & $\sim 0.11$ & 0.1 & $2.4 \times 10^{-3}$ \\
${\text { Reference } 3^{\mathrm{a}}}_{\text {Reference } 4^{\mathrm{a}}}$ & $\sim 0.16$ & Not reported & Not reported \\
${\text { Reference } 9^{\mathrm{a}}}_{\text {This work }}^{\mathrm{b}}$ & $\sim 0.016$ & 0.1 & 0.01 \\
l $^{\mathrm{b}}$ & $\sim 0.04$ & $\sim 0.16$ & $2.9 \times 10^{-3}$ \\
\hline \hline
\end{tabular}

${ }^{a}$ Measurement result.

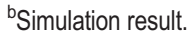

In conclusion, we proposed and demonstrated a method of the wavefront control to achieve polarization insensitivity and broad-band operation based on off-resonance waveguiding. As a proof of concept, we showed a polarization insensitive dielectric microlens operating between 4.0 and $4.6 \mu \mathrm{m}$ with $>94 \%$ relative and $>67 \%$ absolute efficiencies. The proposed method can be applied to every desired wavelength range via a proper choice of the core material. Achieving polarization insensitive broad-band operation with high efficiency in the mid-wave range is a critical step towards implementing the metasurface concept for practical applications in integrated optics in the infrared.

See supplementary material for electric field distribution of a unit cell.

\section{REFERENCES}

'A. Arbabi, Y. Horie, M. Bagheri, and A. Faraon, Nat. Nanotechnol. 10, 937 (2015).

${ }^{2}$ A. Arbabi, Y. Horie, A. J. Ball, M. Bagheri, and A. Faraon, Nat. Commun. 6, 7069 (2015).

${ }^{3}$ M. Khorasaninejad, W. T. Chen, R. C. Devlin, J. Oh, A. Y. Zhu, and F. Capasso, Science 352(6290), 1190 (2016).

${ }^{4}$ W. T. Chen, A. Y. Zhu, V. Sanjeev, M. Khorasaninejad, Z. Shi, E. Lee, and F. Capasso, Nat. Nanotechnol. 13, 220 (2018).

${ }^{5}$ M. Khorasaninejad, A. Y. Zhu, C. Roques-Carmes, W. T. Chen, J. Oh, I. Mishra, R. C. Devlin, and F. Capasso, Nano Lett. 16, 7229 (2016).

${ }^{6}$ O. Akin and H. V. Demir, Appl. Phys. Lett. 110, 143106 (2017).

${ }^{7}$ E. Arbabi, A. Arbabi, S. M. Kamali, Y. Horie, and A. Faraon, Optica 3, 628 (2016).

${ }^{8}$ M. Khorasaninejad, F. Aieta, P. Kanhaiya, M. A. Kats, P. Genevet, D. Rousso, and F. Capasso, Nano Lett. 15, 5358 (2015).

${ }^{9}$ S. Wang, P. C. Wu, V. C. Su, Y. C. Lai, M. K. Chen, H. Y. Kuo, B. H. Chen, Y. H. Chen, T. T. Huang, J. H. Wang, R. M. Lin, C. H. Kuan, T. Li, Z. Wang, S. Zhu, and D. P. Tsai, Nat. Nanotechnol. 13, 227 (2018).

${ }^{10}$ Z. Zhang, D. Wen, C. Zhang, M. Chen, W. Wang, S. Chen, and X. Chen, ACS Photonics 5, 1794 (2018).

${ }^{1}$ N. K. Emani, E. Khaidarov, R. Paniagua-Domínguez, Y. H. Fu, V. Valuckas, S. Lu, X. Zhang, S. T. Tan, H. V. Demir, and A. I. Kuznetsov, Appl. Phys. Lett. 111, 221101 (2017).

${ }^{12}$ Z. Zhou, J. Li, R. Su, B. Yao, H. Fang, K. Li, L. Zhou, J. Liu, D. Stellinga, C. P. Reardon, T. F. Krauss, and X. Wang, ACS Photonics 4, 544 (2017).

${ }^{13}$ O. Akın and H. V. Demir, Opt. Express 23, 27020 (2015).

${ }^{14}$ J. Hu, C. H. Liu, X. Ren, L. J. Lauhon, and T. W. Odom, ACS Nano 10, 10275 (2016).

${ }^{15}$ Y. F. Yu, A. Y. Zhu, R. Paniagua-Domínguez, Y. H. Fu, B. Luk'yanchuk, and A. I. Kuznetsov, Laser Photonics Rev. 9, 412 (2015).

${ }^{16} \mathrm{M}$. Khorasaninejad, W. Zhu, and K. B. Crozier, Optica 2, 376 (2015).

${ }^{17}$ A. Arbabi, R. M. Briggs, Y. Horie, M. Bagheri, and A. Faraon, Opt. Express 23, 33310 (2015).

${ }^{18}$ M. Khorasaninejad, Z. Shi, A. Y. Zhu, W. T. Chen, V. Sanjeev, A. Zaidi, and F. Capasso, Nano Lett. 17, 1819 (2017).

${ }^{19} \mathrm{M}$. Khorasaninejad and F. Capasso, Nano Lett. 15, 6709 (2015).

${ }^{20}$ R. Paniagua-Domínguez, Y. F. Yu, E. Khaidarov, S. Choi, V. Leong, R. M. Bakker, X. Liang, Y. H. Fu, V. Valuckas, L. A. Krivitsky, and A. I. Kuznetsov, Nano Lett. 18 2124 (2018).

${ }^{21}$ S. M. Kamali, E. Arbabi, A. Arbabi, Y. Horie, and A. Faraon, Laser Photonics Rev. 10, 1002 (2016).

${ }^{22}$ A. Arbabi, E. Arbabi, Y. Horie, S. M. Kamali, S. Han, and A. Faraon, "Aberration corrected metasurface doublet lens," in Conference on Lasers and ElectroOptics, OSA Technical Digest (2016) (Optical Society of America, 2016), paper STh1E.6.

${ }^{23}$ F. Aieta, M. A. Kats, P. Genevet, and F. Capasso, Science 347, 6228 (2015).

${ }^{24}$ A. Yariv and P. Yeh, Photonics: Optical Electronics in Modern Communications (Oxford University Press, New York, NY, 2009), pp. 802-811.

${ }^{25}$ Q. Zhao, J. Zhou, F. Zhang, and D. Lippens, Mater. Today 12, 60 (2009).

${ }^{26}$ P. Genevet, F. Capasso, F. Aieta, M. Khorasaninejad, and R. Devlin, Optica 4, 139 (2017). 